\title{
Simultaneous high expression of PLD1 and Sp1 predicts a poor prognosis for pancreatic ductal adenocarcinoma patients
}

\author{
Jiong Hu ${ }^{1,2,3, *}$, Hai Hu ${ }^{2,3, *}$, Jun-jie Hang ${ }^{2,3, *}$, Hai-yan Yang ${ }^{2,3, *}$, Zhi-yong Wang ${ }^{2,3}$, Lei \\ Wang ${ }^{2,3}$, Dong-hui Chen ${ }^{2,3}$, Li-wei Wang ${ }^{1,2,3}$ \\ ${ }^{1}$ Department of Medical Oncology, Shanghai General Hospital of Nanjing Medical University, Shanghai 201620, China \\ ${ }^{2}$ Department of Medical Oncology and Pancreatic Cancer Center, Shanghai General Hospital, Shanghai Jiao Tong University \\ School of Medicine, Shanghai 201620, China \\ ${ }^{3}$ Shanghai Key Laboratory of Pancreatic Diseases, Shanghai 201620, China \\ *These authors contributed equally to this work
}

Correspondence to: Dong-hui Chen, email: chendh100@163.com

Li-wei Wang, email: yzwlw@hotmail.com

Keywords: PDAC, PLD1, Sp1, prognosis, immunohistochemistry

Received: March 09, $2016 \quad$ Accepted: September 20, 2016

Published: October 04, 2016

\section{ABSTRACT}

Pancreatic ductal adenocarcinoma (PDAC) is a lethal disease with few therapeutic options. Recently, insight into cancer biology suggested abnormal lipid metabolism to be a risk factor for human malignancies. As a key enzyme implicated in lipid metabolism, PLD1 was elevated in various human cancer associating with malignant phenotypes. However, little was known about its expression and function in PDAC. We showed that PLD1 was elevated in both the cell lines and clinical samples of PDAC, and it positively correlated with vascular invasion $(p=0.041)$ and responsible for a poor prognosis $(p=0.009)$. Meanwhile, we also found Sp1 to be elevated in the disease, correlating with vascular invasion $(p=0.007)$. Moreover, the correlation assay suggested that PLD1 positively correlated with Sp1 in the clinical sample $(r=0.390$; $p<0.001$ ) and the cell lines. Finally, we showed that co-high expression of both the factors confers the poorest prognosis for the patients, and that their simultaneous high expression might be an independent prognostic factor $(p=0.001 ; H R=3.427$; 95\% CI 1.629-7.211).

\section{INTRODUCTION}

Pancreatic ductal adenocarcinoma (PDAC) is a lethal disease with the 5-year survival rate of less than 5\% and the median survival of about 6 months, rendering it the fourth most lethal cancer in the United States [1], a frustrating situation had not been changed for decades. Major causes for the disappointing situation include high propensity of early distant metastasis and chemoresistence $[2,3]$. However, the molecular and cellular mechanisms underlying these processes remain illusive.

Recently, mounting evidence had suggested abnormal lipid metabolism of the cancer cells to be protumoral in various human cancers [4]. PLD1 was a key enzyme implicated in lipid metabolism by catalyzing the hydrolysis of phosphatidylcholine. As stated, PLD1was elevated in most human malignancies associating with malignant phenotypes. For instance, the prior studies indicated that PLD1 was upregulated in cancers of the intestinal [5] and breast [6]. Functionality analysis showed that elevated PLD1 had a positive correlation with angiogenesis, invasion and distant metastasis as well as chemoresistence of human cancer [7, 8]. Despite of the advancement, little was known about its expression and biological significance in PDAC.

$\mathrm{Sp} 1$ is a basal transcription factor belonging to the krüppel-like factor family, and it expressed in nearly all cells of an individual and responsible for proliferation, division, and differentiation [9, 10]. As to cancer, Sp1 was found to be elevated in most tumors responsible for unfavorable phenotypes via transcription activation $[11,12]$. For stance, it had been reported that elevated Sp1 contributes to overexpression of multiple oncogenic genes in human cancers [13], including PDAC [14]. For example, Bae IH and colleagues showed that Bcl-w promotes gastric cancer cell invasion by inducing matrix metalloproteinase-2 expression via phosphoinositides 3-kinase, Akt, and Sp1 [15]. Consistently, there was 
also report that Celecoxib inhibits VEGF expression and reduces angiogenesis and metastasis of pancreatic cancer via the suppression of Sp1 [14]. Since PLD1 was also reported as an oncogenic gene in various human cancers, we boldly postulated that it positive correlated with $\mathrm{Sp} 1$, and they could promote PDAC progression synergistically.

In the present study, the expression and the biological significance of PLD1 were investigated. We showed that PLD1 was elevated in PDAC, and it positively correlated with vascular invasion and poor survival. Meanwhile, we also showed that Sp1 was elevated in PDAC, and it significantly correlated with vascular invasion. Moreover, we also showed that PLD1 positively correlated with Sp1 in PDAC, and their simultaneous high expression was an independent prognostic factor for the patients.

\section{RESULTS}

\section{The baseline characteristics of the PDAC patients}

The baseline clinicopathlogical characteristics of the PDAC patients enrolled in this study are summarized in Table 1 . Of the 77 patients, 51 were male and 26 were female. The median age of the patients was 62 (ranged from 20 to 78 ). 73 patients were stage I and/or II, while the rest were diagnosed as metastatic disease. 40 patients had cancers of the head and neck of pancreas, while 37 had cancers in the body and tail of pancreas. Notably, 49 patients exhibited nerve invasion and 10 patients showed vascular invasion.

\section{PLD1 was elevated in pancreatic cancer}

To examine the biological significance of PLD1 in PDAC, IHC were used to determine its expression in the tumors. We found that their staining in the patients ranged from weak to strong (Figure 1A). Additionally, we also found a significant difference of PLD1 expression between the cancerous tissues and the paired normal tissues (Figure 1B). Subsequently, we differentiated PLD1 positive patients from their negative counterparts and found that half of the patients were PLD1 positive (Figure 3). Moreover, the correlation assay showed that PLD1 was significantly higher in patients with vascular invasion ( $p=0.041)$ compared to those without vascular invasion (Table 2). However, no obvious significance could be observed with other parameters of the patients. Finally, we showed in the survival analysis that PLD1 indicated a poor prognosis ( $p=0.021$, Figure $1 C$ ).

\section{Sp1 was elevated in pancreatic cancer}

Meanwhile, we also investigated Sp1 expression in PDAC using IHC. As shown in Figure 2A, we found that the staining of Sp1 ranged from negative to strong, with half the patients positive were positive PLD1 staining (Figure 3). Additionally, we also showed a significant difference of Sp1 staining between the cancerous tissues and the paired none cancerous tissues (Figure 2B). Furthermore, the correlation assay showed that $\mathrm{Sp} 1$ positively correlated with vascular invasion $(p=0.007$, Table 2); while no obvious significance could be observed with other parameters of the patients. Finally, the survival analysis showed that $\mathrm{Sp} 1$ also confers a poor prognosis for the patients ( $p=0.012$, Figure $2 \mathrm{C}$ ).

\section{Correlation between PLD1 and Sp1 in PDAC tissues}

Since both $\mathrm{Sp} 1$ and PLD1 contribute to the aggressive of various human cancers, we postulated that they were correlated in the disease. To attain this, serial sections of the same PDAC tissues were scored for stained Sp1 and PLD1 respectively. The final scores of the patients were used to conduct correlation assay, and the data showed that $\mathrm{Sp} 1$ is positively correlated with PLD1 $(r=0.390 ; p<0.001$, Figure 4A). Moreover, we showed in the Kaplan-Meier assay that combined expression of the two factors confers the poorest prognosis among all the patients $(p=0.001$, Figure $4 \mathrm{~B}, 4 \mathrm{C})$. Finally, we showed in the multivariate analysis that the combined high expression of Sp1 and PLD1 was an independent prognostic factor for the patients $(p=0.001$; $\mathrm{HR}=3.427$; 95\% CI 1.629-7.211, Table 3).

Additionally, we also found in the multivariate analysis that TNM stage $(p=0.042 ; \mathrm{HR}=3.223$; $95 \%$ CI 1.044-9.951), and nuclear grade $(p=0.003$; $\mathrm{HR}=2.725 ; 95 \%$ CI 1.391-5.341) were independent prognostic factors for PDAC patients (Table 3 ).

\section{Correlation between PLD1 and Sp1 in PDAC cell lines}

To further confirm the positive correlation between in Sp1 and PLD1 in PDAC, we investigated their expression and correlation in pancreatic duct epithelial (HPDE) cells and pancreatic cancer cell. As shown in Figure 4D, we found that both Sp1 and PLD1 were elevated in cancer cell lines compared to HPDE. Subsequently, we knockout Sp1 expression of the cancer cells and found that PLD1 decreased concomitantly (Figure 4E). Taken together, our data showed that Sp1 positive correlated with PLD1 in PDAC.

\section{DISCUSSION}

There is growing interest in understanding the role that abnormal metabolism in the initiation and progression of cancer. In this study, we had focused on the role of PLD1, a key enzyme implicated in lipid metabolism, in PDAC. Our results show that PLD1 was elevated in the disease and 
Table 1: The baseline characteristics of PDAC patients

\begin{tabular}{|c|c|c|}
\hline Characteristics & Categories & Number (\%) \\
\hline \multirow[t]{2}{*}{ Gender } & Male & $51(66.2)$ \\
\hline & Female & $26(33.8)$ \\
\hline Age median (range) & & $62(20-78)$ \\
\hline \multirow[t]{3}{*}{ T stage } & $\mathrm{T} 1$ & $4(5.2)$ \\
\hline & $\mathrm{T} 2$ & $16(20.8)$ \\
\hline & T3 & $57(74.0)$ \\
\hline \multirow[t]{2}{*}{ N stage } & No & $35(45.5)$ \\
\hline & N1 & $42(54.5)$ \\
\hline \multirow[t]{2}{*}{ M stage } & M0 & $73(94.8)$ \\
\hline & M1 & $4(5.2)$ \\
\hline \multirow[t]{5}{*}{$\mathrm{TMN}$} & IA & $4(5.2)$ \\
\hline & IB & $10(13.0)$ \\
\hline & IIA & $20(26.0)$ \\
\hline & IIB & $39(50.6)$ \\
\hline & IV & $4(5.2)$ \\
\hline \multirow[t]{2}{*}{ Primary tumor location } & Head and Neck & $40(51.9)$ \\
\hline & Body and Tail & $37(48.1)$ \\
\hline \multirow[t]{3}{*}{ Nuclear grade } & $\mathrm{I}$ & $9(11.7)$ \\
\hline & II & $42(54.5)$ \\
\hline & III & $26(33.8)$ \\
\hline \multirow[t]{2}{*}{ Nerve invasion } & Yes & $49(63.6)$ \\
\hline & No & $28(36.4)$ \\
\hline \multirow[t]{2}{*}{ Vascular invasion } & Yes & $10(13.0)$ \\
\hline & No & $67(87.0)$ \\
\hline
\end{tabular}

it correlated with vascular invasion. More importantly, our data further showed that Sp1 is elevated in PDAC, and it positively correlated with PLD1 with that their simultaneous overexpression predicts a poor prognosis for patients.

PLD1 functions to catalyze the hydrolysis of phosphatidylcholine PC so as to generate phosphatidic acid (PA), substrates associating with various signaling cascades, such as Wnt, mTOR, and NF-kB [7, 16, 17]. As stated, PLD1 were reported to be overexpression in various human tumors and contribute to the malignant phenotypes of human cancers, such as angiogenesis, invasion and metastasis, and chemoresistence $[8,18,19]$.

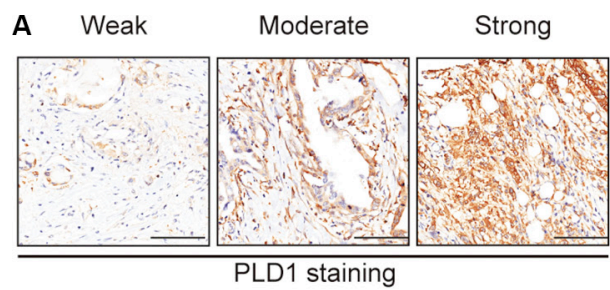

PLD1 staining
B

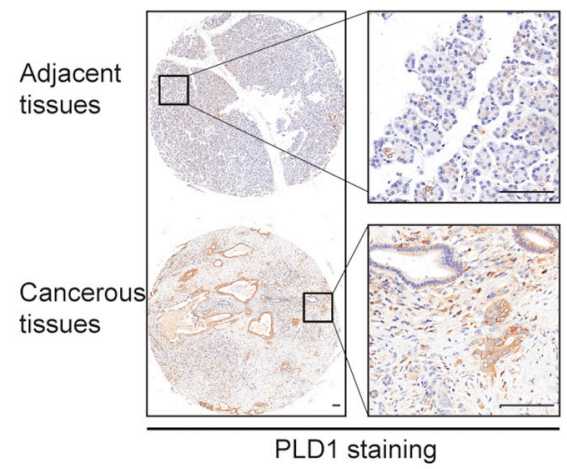

C

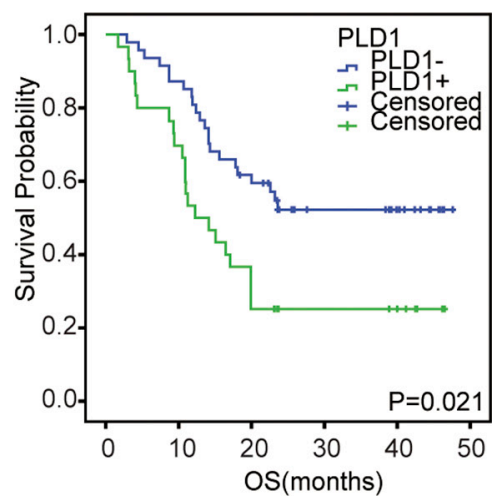

Figure 1: Representative immunohistochemical staining of Sp1 (A-C) and PLD1 (D-E) in PDAC. (A, D): Weak positive staining; (B, E): Moderate positive staining; (C, F): Strong positive staining. Bar: $100 \mathrm{um}$. 
Table 2: Correlation between Sp1, PLD1 and clinicopathologic features of PDAC patients

\begin{tabular}{|c|c|c|c|c|c|c|c|}
\hline \multirow[b]{2}{*}{ Factor } & \multirow[b]{2}{*}{ Number } & \multicolumn{3}{|c|}{ Sp1 } & \multicolumn{3}{|c|}{ PLD1 } \\
\hline & & Negative & Positive & $P$ & Negative & Positive & $P$ \\
\hline \multicolumn{8}{|l|}{ Gender } \\
\hline Male & 51 & 27 (52.9) & $24(47.1)$ & 0.573 & $31(60.8)$ & $20(39.2)$ & 0.949 \\
\hline Female & 26 & $12(46.2)$ & $14(53.8)$ & & $16(61.5)$ & $10(38.5)$ & \\
\hline \multicolumn{8}{|l|}{ Age } \\
\hline$>60$ & 51 & 27 (52.9) & $24(47.1)$ & 0.573 & $30(58.8)$ & $21(41.2)$ & 0.577 \\
\hline$<60$ & 26 & $12(46.2)$ & $14(53.8)$ & & $17(65.4)$ & $9(34.6)$ & \\
\hline \multicolumn{8}{|l|}{ T stage } \\
\hline T3 & 57 & $29(50.9)$ & $28(49.1)$ & 0.946 & 37 (64.9) & $20(35.1)$ & 0.239 \\
\hline$<\mathrm{T} 2$ & 20 & $10(50.0)$ & $10(50.0)$ & & $10(50.0)$ & $10(50.0)$ & \\
\hline \multicolumn{8}{|l|}{ N stage } \\
\hline N0 & 35 & $20(57.1)$ & $15(42.9)$ & 0.298 & $23(65.7)$ & $12(34.3)$ & 0.443 \\
\hline N1 & 42 & $19(45.2)$ & $23(54.8)$ & & $24(57.1)$ & $18(42.9)$ & \\
\hline \multicolumn{8}{|l|}{ M stage } \\
\hline M0 & 73 & $37(50.7)$ & $36(49.3)$ & 0.979 & $44(60.3)$ & $29(39.7)$ & 0.951 \\
\hline M1 & 4 & $2(50.0)$ & $2(50.0)$ & & $3(75.0)$ & $1(25.0)$ & \\
\hline \multicolumn{8}{|l|}{ TMN stage } \\
\hline$>$ II & 63 & 31 (49.2) & $32(50.8)$ & 0.591 & 39 (61.9) & $24(38.1)$ & 0.741 \\
\hline I & 14 & $8(57.1)$ & $6(42.9)$ & & $8(57.1)$ & $6(42.9)$ & \\
\hline \multicolumn{8}{|c|}{ Primary tumor location } \\
\hline Head and Neck & 40 & $17(42.5)$ & $23(57.5)$ & 0.137 & $23(42.5)$ & $17(57.5)$ & 0.508 \\
\hline Body and Tail & 37 & $22(59.5)$ & $15(40.5)$ & & $24(64.9)$ & $13(35.1)$ & \\
\hline \multicolumn{8}{|l|}{ Nerve invasion } \\
\hline Yes & 49 & $24(49.0)$ & $25(51.0)$ & 0.698 & $30(61.2)$ & $19(38.8)$ & 0.965 \\
\hline No & 28 & $15(53.6)$ & $13(46.4)$ & & $17(60.7)$ & $11(39.3)$ & \\
\hline \multicolumn{8}{|l|}{ Vascular invasion } \\
\hline Yes & 10 & $1(10.0)$ & $9(90.0)$ & 0.007 & $3(30.0)$ & $7(70.0)$ & 0.041 \\
\hline No & 67 & $38(56.7)$ & $29(43.3)$ & & $44(65.7)$ & $23(34.3)$ & \\
\hline \multicolumn{8}{|l|}{ Nuclear grade } \\
\hline III & 26 & $11(42.3)$ & $15(57.7)$ & 0.296 & $13(50.0)$ & $13(50.0)$ & 0.156 \\
\hline$<$ II & 51 & $28(54.9)$ & $23(45.1)$ & & $34(66.7)$ & $17(33.3)$ & \\
\hline
\end{tabular}

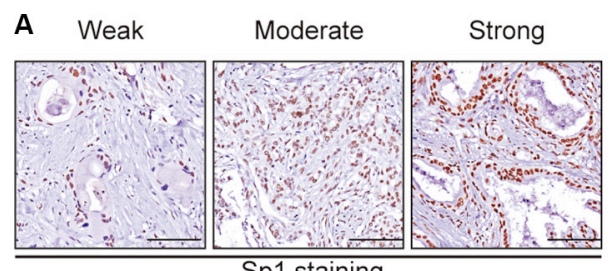

B

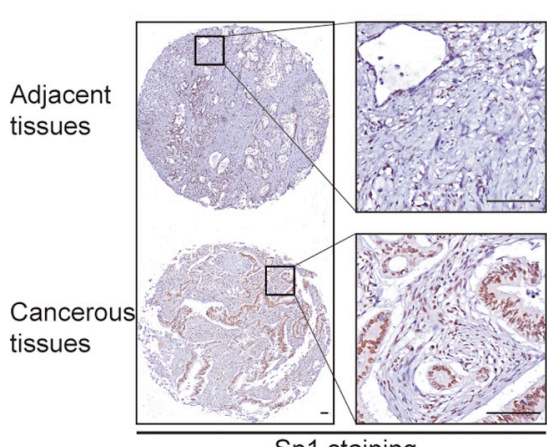

C

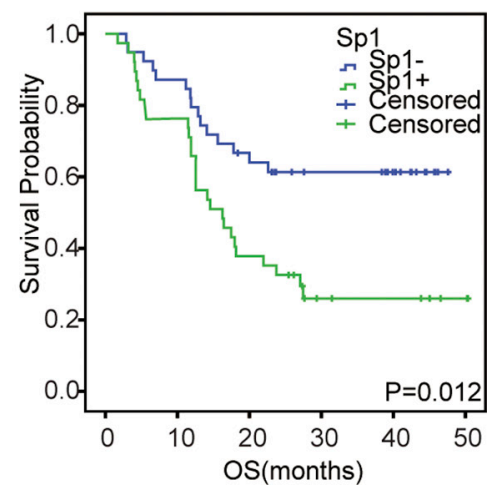

Figure 2: The expression pattern of Sp1 and PLD1 in PDAC. (A): The expression pattern of Sp1 and PLD1 based on intensity and percentage of the stained cells in PDAC. (B) Representative images of PDAC tissues with positive/negative Sp1 and PLD1 expression. Bar: $100 \mu \mathrm{m}$. 
In the present study, we reported for the first time that PLD1 is overexpression in PDAC. Our data also revealed that PLD1 is closely correlated with vascular invasion, suggesting that PLD1 might also involve in local invasion and distant metastasis of PDAC. Taken together, the data highlights a critical role of PLD1 in PDAC, and that targeted inhibition of PLD1 might be a novel direction for the management of PDAC.

$\mathrm{Sp} 1$ is a ubiquitously expressed nuclear transcription factor with three zinc fingers in the $\mathrm{C}$-terminal domains that binds to the GC/GT box of target genes [20]. Under normal conditions, $\mathrm{Sp} 1$ was expressed in all cells of an individual responsible for cell propagation, differentiation and division. As stated, it is also overexpression in most human cancers and functions to stimulate angiogenesis, invasion and metastasis as well as chemoresistence by upregulating the relevant genes expression [21-23]. For instance, Xie and colleagues [24] found that Sp1 could transcriptionally activate VEGF expression, a molecule associates with angiogenesis and distant metastasis in PDAC. Moreover, some researchers proposed early in 1993 that Sp1 was responsible for chemoresistence; since they showed that Sp1 could transcriptionally activate MDR expression [25]. Consistently, our data also showed that $\mathrm{Sp} 1$ was positively correlated with vascular invasion in PDAC, suggesting that it might also involved in distant metastasis of PDAC.

Previously, researchers had established the positive correlation for Sp1 and other genes, they showed that there was a zinc finger in the C-terminal of Sp1, and it is the very structure that binds to the $\mathrm{GC} / \mathrm{GT}$ boxes of the target genes leading to their expression [26]. In the present study, we showed that $\mathrm{Sp} 1$ positively correlated with PLD1, and that their simultaneous high expression confers the poorest prognosis for the patients. These data showed that the two
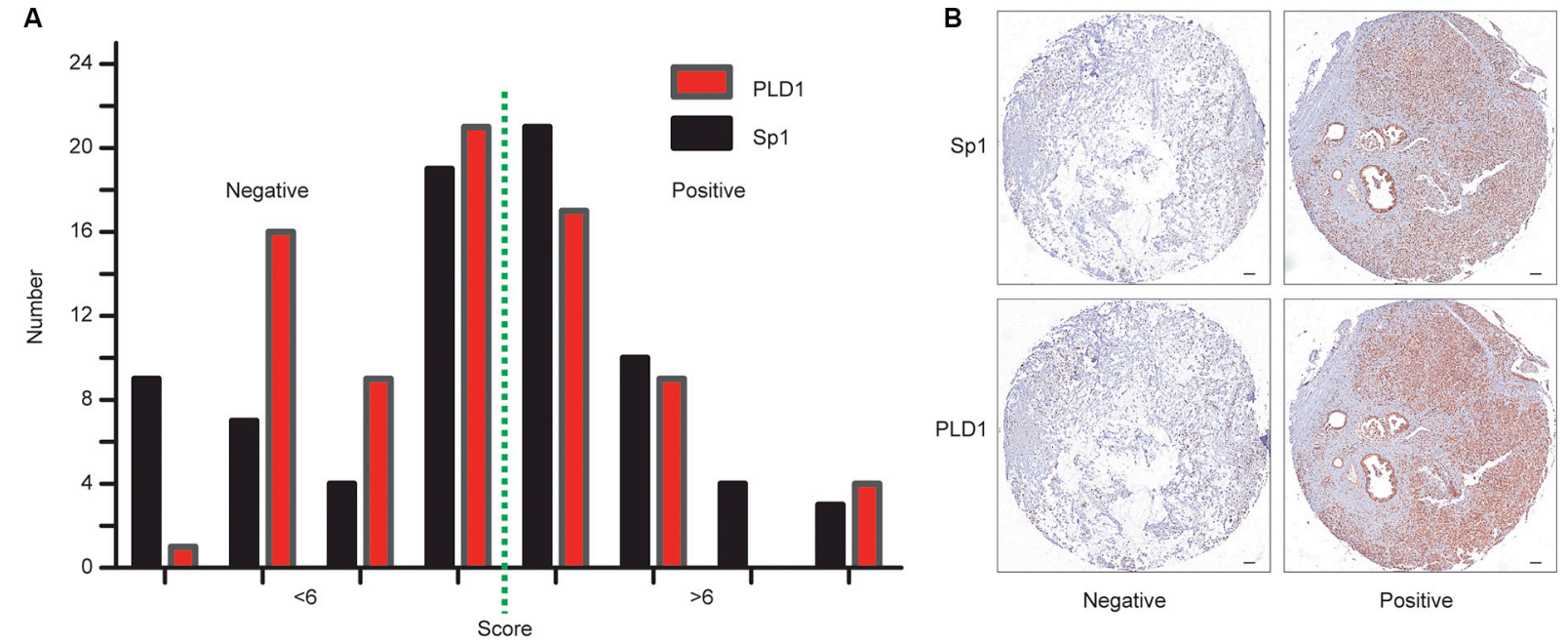

Figure 3: Correlation between Sp1 and PLD1 expression in PDAC samples and cell lines. (A) The picture depicted the co-distribution of Sp1 and PLD1 in PDAC samples. Spearman's rank correlation coefficient demonstrated a significant correlation between Sp1 and PLD1 $(r=0.390 ; P<0.001)$. (B) showed the PLD1 expression upon Sp1 deletion.

A

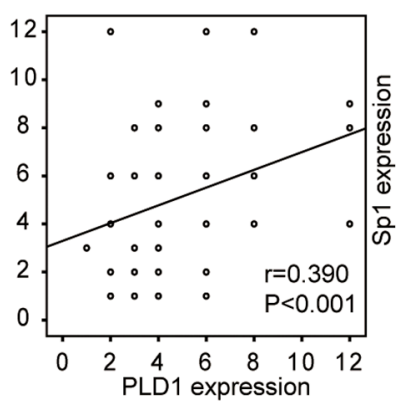

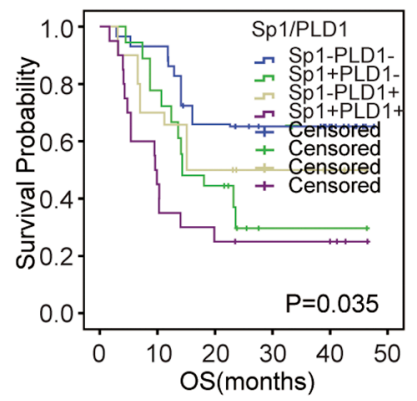

C

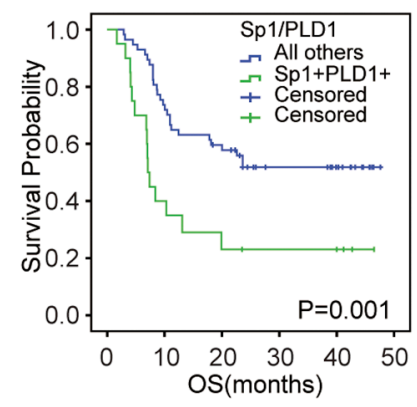

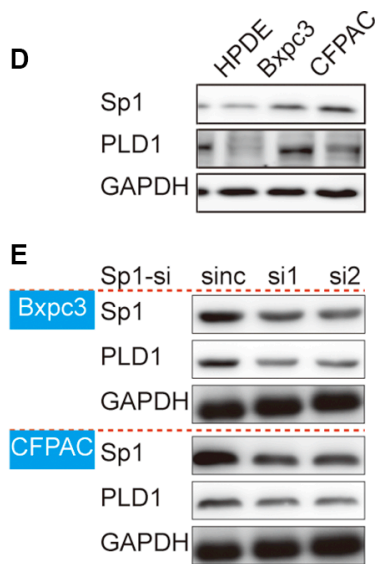

Figure 4: Overall survival curves based on Sp1 and PLD1 expression in PDAC. The overall survival curves were based on Sp1 (A), PLD1 (B), and the combination of Sp1 and PLD1 (C and D) (as polytomous variables and binary categorical variables, respectively). All others: $\mathrm{Sp} 1^{+} \mathrm{PLD}_{1}{ }^{-}, \mathrm{Sp} 1^{-} \mathrm{PLD}^{+}$, and $\mathrm{Sp} 1^{-} \mathrm{PLD} 1^{-}$. 
Table 3: Univariate and multivariate survival analysis of clinicopathologic variables in PDAC patients

\begin{tabular}{|c|c|c|c|c|c|c|c|}
\hline \multirow{2}{*}{ Factor } & \multirow{2}{*}{$\begin{array}{l}\text { OS median } \\
\text { (range) }\end{array}$} & \multicolumn{3}{|c|}{ Univariate analysis } & \multicolumn{3}{|c|}{ Multivariate analysis } \\
\hline & & HR & $95 \%$ CI & $P$ & HR & $95 \%$ CI & $P$ \\
\hline \multicolumn{8}{|l|}{ Gender } \\
\hline Male & $19.0(1.0-47.0)$ & 1.054 & $0.555-2.004$ & 0.871 & & & \\
\hline Female & $20.0(3.0-46.0)$ & 1 & & & & & \\
\hline \multicolumn{8}{|l|}{ Age } \\
\hline$>60$ & $22.0(1.0-47.0)$ & 0.677 & $0.365-1.254$ & 0.215 & & & \\
\hline$<60$ & $15.5(2.0-46.0)$ & 1 & & & & & \\
\hline \multicolumn{8}{|l|}{ T stage } \\
\hline $\mathrm{T} 3$ & $17.8(1.7-47.6)$ & 1.778 & $0.822-3.845$ & 0.144 & & & \\
\hline$<\mathrm{T} 2$ & $23.0(3.0-46.0)$ & 1 & & & & & \\
\hline \multicolumn{8}{|l|}{$\mathrm{N}$ stage } \\
\hline N0 & $22.3(2.9-47.6)$ & 1.279 & $0.694-2.357$ & 0.431 & & & \\
\hline N1 & 17. $(1.7-45.8)$ & 1 & & & & & \\
\hline \multicolumn{8}{|l|}{ M stage } \\
\hline M0 & $18.4(1.7-47.6)$ & 0.658 & $0.175-3.005$ & 0.658 & & & \\
\hline M1 & $23.2(12.4-40.3)$ & 1 & & & & & \\
\hline \multicolumn{8}{|l|}{ TMN stage } \\
\hline$>$ II & $15.6(1.7-47.6)$ & 3.052 & $1.087-8.567$ & 0.034 & 3.223 & $1.044-9.951$ & 0.042 \\
\hline I & $31.1(12.6-46.5)$ & 1 & & & 1 & & \\
\hline \multicolumn{8}{|c|}{ Primary tumor location } \\
\hline $\begin{array}{l}\text { Head and } \\
\text { Neck }\end{array}$ & $16.4(1.7-47.6)$ & 1.204 & $0.657-2.209$ & 0.548 & & & \\
\hline Body and Tail & $23.1(2.9-46.4)$ & 1 & & & & & \\
\hline \multicolumn{8}{|l|}{$\begin{array}{l}\text { Nerve } \\
\text { invasion }\end{array}$} \\
\hline Yes & $13.6(1.7-46.5)$ & 2.171 & $1.089-4.328$ & 0.028 & 1.573 & $0.738-3.356$ & 0.241 \\
\hline No & $23.7(3.1-47.6)$ & 1 & & & 1 & & \\
\hline \multicolumn{8}{|l|}{$\begin{array}{l}\text { Vascular } \\
\text { invasion }\end{array}$} \\
\hline Yes & $7.9(3.1-46.5)$ & 2.958 & $1.352-6.473$ & 0.007 & 2.560 & $0.977-6.705$ & 0.056 \\
\hline No & $22.3(1.7-47.6)$ & 1 & & & 1 & & \\
\hline \multicolumn{8}{|l|}{ Nuclear grade } \\
\hline III & $12.1(1.7-44.6)$ & 2.374 & $1.290-4.368$ & 0.005 & 2.725 & $1.391-5.341$ & 0.003 \\
\hline$<$ II & $23.2(4.0-47.6)$ & 1 & & & 1 & & \\
\hline \multicolumn{8}{|l|}{ Sp1 } \\
\hline Positive & $12.5(1.7-46.5)$ & 2.599 & $1.377-4.903$ & 0.003 & & & \\
\hline Negative & $23.7(2.9-47.6)$ & 1 & & & & & \\
\hline \multicolumn{8}{|l|}{ PLD1 } \\
\hline Positive & $9.4(1.7-46.5)$ & 2.212 & $1.203-4.069$ & 0.011 & & & \\
\hline Negative & $23.2(2.9-47.6)$ & 1 & & & & & \\
\hline \multicolumn{8}{|l|}{ Sp1/PLD1 } \\
\hline Sp1+/PLD1+ & $7.3(1.7-46.5)$ & 2.768 & $1.463-5.238$ & 0.002 & 3.427 & $1.629-7.211$ & 0.001 \\
\hline All others & $23.1(2.9-47.6)$ & 1 & & & 1 & & \\
\hline
\end{tabular}


factors might collaborate with each other so as to promote PDAC progression. Hence, further studies were needed to clarify whether transcription activation also apply to the positive correlations between Sp1 and PLD1 in PDAC. In addition, some of the prior studies indicated that PLD1 functions upstream of Wnt/beta-catenin [27] and JAK/ STAT3 [28] signaling, which subsequently phosphorylate the downstream molecules. As phosphorylated Sp1 was the active form of Sp1; we, therefore, proposed another hypothesis to link them together, which suggested that PLD1-triggered signaling could subsequently activate Sp1 by phosphorylation in PDAC.

In conclusion, our findings have revealed strong expression of PLD1 and Sp1 in PDAC. In addition, our data showed that Sp1 positively correlated with PLD1 in the lethal disease. Statistical analysis demonstrated that both $\mathrm{Sp} 1$ and PLD1 correlated closely with vascular invasion and their simultaneous overexpression confers a poor prognosis for patients. Since PLD1 serves as an oncogenic protein in PDAC, further studies are needed to determine its full function and regulation so as to reveal novel therapeutic targets for the lethal disease.

\section{MATERIALS AND METHODS}

\section{Patients}

77 patients with histopathologic diagnosis of primary PDAC (ICD, Tenth Revision, codes C25) were included in our study. Pancreatic cancerous tissues and adjacent paired normal tissues were collected from the department of pathology at Shanghai Jiaotong University Affiliated Shanghai General Hospital, Shanghai, China from 2012 to 2014. The last follow-up visit was on February 28th, 2016. The patients' clinicopathological characteristics include age, gender, TNM stage, primary tumor location, nerve invasion, vascular invasion, and nuclear grade (Table 1). Each patient provided written informed consent and the study approved Ethics Committees of Shanghai General Hospital.

\section{Tissue microarray construction}

The microarray was made as described [29]. Briefly, H\&E-stained sections were made from primary tumor blocks to define tumor regions. Representative tumor regions are defined as areas with at least $75 \%$ cancer cells without necrosis. Tissue cylinders (1.5 $\mathrm{mm}$ in diameter) were then punched from the regions of the block using a tissue microarrayer (Gentury, IL, USA) and placed into recipient paraffin blocks. Sections of the TMA blocks were transferred to glass slides.

\section{Immunohistochemistry (IHC)}

The standard IHC protocol has been described previously [21]. In brief, the tissue microarrays were dewaxed and dehydrated in xylene and alcohol bath solutions, respectively. Endogenous peroxidase activity was then blocked using $0.3 \%$ hydrogen peroxide for 10 mins, before antigen retrieval was undertaken by setting the slides in $0.01 \mathrm{M}$ citrate buffer $\left(\mathrm{pH} \mathrm{6.0)}\right.$ ) at $98^{\circ} \mathrm{C}$ for $5 \mathrm{~min}$ using a microwave oven. The slides were cooled to room temperature and blocked by incubating them with normal goat serum at room temperature for $1 \mathrm{~h}$, followed by incubation at $4{ }^{\circ} \mathrm{C}$ overnight with the primary antibodies (Cell Signaling Technology, Beverly, MA, USA). Finally, the sections were incubated with HRP-labeled secondary antibody and visualized using diaminobenzidine.

\section{Evaluation of IHC}

Evaluation of the staining was performed by two independent pathologists blind to research in at five areas at $400 \times$ magnification. The staining was scored according to the intensity and percentage of the stained cells. Staining intensity was assigned as 0 (no staining), 1 (weak staining), 2 (moderate staining), and 3 (strong staining). The percentages were classified into: $1(\leq 25 \%), 2(25 \%-50 \%)$, $3(50 \%-75 \%)$, and $4(75 \%-100 \%)$. The final scores were calculated as the staining intensity $\times$ the percentage of positive cells. For statistical analyses, a score $<6$ was regarded as negative expression, and $>6$ as positive expression. The dilution of the primary antibodies: Sp1 (1:100); PLD1 (1:100).

\section{Cell lines and cell culture}

Human pancreatic duct epithelial (HPDE) cells and pancreatic cancer cell lines were purchased from Shanghai Institute for Life Science, Chinese Academy of Sciences. All the cells were cultured in RPMI 1640 supplemented with 10\% fetal bovine serum (FBS, Gibco, Carlsbad, CA, USA) at $37^{\circ} \mathrm{C}$ in a humidified atmosphere of $95 \%$ air and $5 \% \mathrm{CO} 2$, and grown in a humidified atmosphere of air/ CO2 (95\%: 5\%). Cells with gene deletion/overexpression were cultured in the same condition with $1.5-\mu \mathrm{g} / \mathrm{mL}$ puromycin (Sigma-Aldrich, St. Louis, MO, USA).

\section{Establishment of PDAC cell lines with short hairpin RNA (shRNA)}

The GV-248 lentiviral RNAi expression system (Genechem, Shanghai, China) was used to prepare the lentivirus expressing human Sp1 shRNA. Sp1-expression plasmids were constructed by cloning the cDNA encoding Sp1 into the system. The targeting sequences were, Sp1Si1: 5'-GCAGTACCAATGGCAGCAATG-3'; Sp1-Si2: 5'-GCAGACCTTTACAACTCAA-3'. The scramble sequences was 5'-TTCTCCGAACGTGTCACGT-3'. The polyclonal cells with puromycin resistance were selected for subsequent experiments. The protocol of transfection had been described previously [30]. 


\section{Western blot analysis}

Cells were washed three times with cold PBS and lysed on ice in RIPA buffer with protease inhibitors PMSF (Beyotime Biotechnology, China). Protein concentrations were determined by BCA method (Beyotime Biotechnology, China). A total of $20 \mu \mathrm{g}$ protein was separated by $10 \%$ SDS-PAGE and electroblotted onto NC membranes using semi-dry blotting apparatus. After blocking in 3\% bovine serum albumin (BSA), the membranes were incubated with the primary antibodies overnight at $4^{\circ} \mathrm{C}$. The membranes were washed and incubated with the secondary antibodies for $1 \mathrm{~h}$ at room temperature on a shaker. The protein bands were visualized using a commercially available enhanced chemiluminesence kit (Thermo Scientific, Hudson, $\mathrm{NH}$, USA). GAPDH were used as control. The primary antibodies used in the study include: Sp1 (1:1000), PLD1 (1: 1000) (CST, Beverly, MA, USA); and GAPDH (Santa Cruz Biotechnology, CA, USA).

\section{Statistical analysis}

Statistical analyses were performed using SPSS software (version 21.0; SPSS Inc, Chicago, IL, USA). The relationships between the clinicopathlogical factors and Sp1/PLD1 expression were investigated using Pearson $\chi^{2}$ test. The Spearman's rank test was used to evaluate their correlation. Kaplan-Meier analysis was used to demonstrate differences in overall survival (OS). The correlation between the prognostic factors and OS was investigated with the Cox regression model. Results were considered statistically significant when $p<0.05$.

\section{Abbreviation}

Pld1 phospholipase D1; Sp1 Specificity protein-1.

\section{ACKNOWLEDGMENTS AND FUNDING}

This study was supported in part by the National Natural Science Foundation of China (Grant no. 81171887 and 91229117), the Program of Shanghai Subject Chief Scientist (Grant no. 12XD1404200), the Shanghai Science and Technology Committee (Grant no. 11DZ1922002).

\section{CONFLICTS OF INTEREST}

The authors declare no conflicts of interests.

\section{REFERENCES}

1. Siegel RL, Miller KD, Jemal A. Cancer statistics, 2015. CA: a cancer journal for clinicians. 2015; 65:5-29.

2. Vincent A, Herman J, Schulick R, Hruban RH, Goggins M. Pancreatic cancer. The Lancet. 2011; 378:607-620.
3. Hidalgo M. Pancreatic cancer. New England Journal of Medicine. 2010; 362:1605-1617.

4. Santos CR, Schulze A. Lipid metabolism in cancer. Febs Journal. 2012; 279:2610-2623.

5. Kang DW, Choi CY, Cho YH, Tian H, Di Paolo G, Choi K-Y. Targeting phospholipase D1 attenuates intestinal tumorigenesis by controlling $\beta$-catenin signaling in cancerinitiating cells. The Journal of Experimental Medicine. 2015; 212:1219-1237.

6. Noh DY, Ahn SJ, Lee RA, Park IA, Kim JH, Suh PG, Ryu SH, Lee KH, Han JS. Overexpression of phospholipase D1 in human breast cancer tissues. Cancer letters. 2000; 161:207-214.

7. Kang DW, Choi KY. Phospholipase D meets Wnt signaling: a new target for cancer therapy. Cancer research. 2011; 71:293-297.

8. Chen Q, Sato T, Hongu T, Zhang Y, Ali W, Cavallo JA, van der Velden A, Tian H, Di Paolo G, Nieswandt B. Key roles for the lipid signaling enzyme phospholipase d1 in the tumor microenvironment during tumor angiogenesis and metastasis. Science signaling. 2012; 5:ra79.

9. Black AR, Black JD, Azizkhan, Clifford J. Sp1 and krüppel, like factor family of transcription factors in cell growth regulation and cancer. Journal of cellular physiology. 2001; 188:143-160.

10. Marin M, Karis A, Visser P, Grosveld F, Philipsen S. Transcription factor $\mathrm{Sp} 1$ is essential for early embryonic development but dispensable for cell growth and differentiation. Cell. 1997; 89:619-628.

11. Li L, Davie JR. The role of Sp1 and Sp3 in normal and cancer cell biology. Annals of Anatomy-Anatomischer Anzeiger. 2010; 192:275-283.

12. Chuang JY, Wu CH, Lai MD, Chang WC, Hung JJ. Overexpression of $\mathrm{Sp} 1$ leads to $\mathrm{p} 53$, dependent apoptosis in cancer cells. International Journal of Cancer. 2009; 125:2066-2076.

13. Chu S, Ferro TJ. Sp1: regulation of gene expression by phosphorylation. Gene. 2005; 348:1-11.

14. Wei D, Wang L, He Y, Xiong HQ, Abbruzzese JL, Xie K. Celecoxib inhibits vascular endothelial growth factor expression in and reduces angiogenesis and metastasis of human pancreatic cancer via suppression of $\mathrm{Sp} 1$ transcription factor activity. Cancer Res. 2004; 64:2030-2038.

15. Bae IH, Park MJ, Yoon SH, Kang SW, Lee SS, Choi KM, Um HD. Bcl-w promotes gastric cancer cell invasion by inducing matrix metalloproteinase-2 expression via phosphoinositide 3-kinase, Akt, and Sp1. Cancer Res. 2006; 66:4991-4995.

16. Kang DW. Platelet derived growth factor increases phospholipase D1 but not phospholipase D2 expression via NFאB signaling pathway and enhances invasion of breast cancer cells. Cancer letters. 2010; 294:125-133. 
17. Fang Y, Park IH, Wu AL, Du G, Huang P, Frohman MA, Walker SJ, Brown HA, Chen J. PLD1 regulates mTOR signaling and mediates Cdc42 activation of S6K1. Current biology. 2003; 13:2037-2044.

18. Kang D, Hwang W, Park M, Ko G, Ha W, Kim K, Lee Y, Choi K, Min D. Rebamipide abolishes Helicobacter pylori CagA-induced phospholipase D1 expression via inhibition of NFKB and suppresses invasion of gastric cancer cells. Oncogene. 2013; 32:3531-3542.

19. Gozgit J, Pentecost B, Marconi S, Ricketts-Loriaux R, Otis C, Arcaro K. PLD1 is overexpressed in an ER-negative MCF-7 cell line variant and a subset of phospho-Aktnegative breast carcinomas. British journal of cancer. 2007; 97:809-817.

20. Kadonaga JT, Carner KR, Masiarz FR, Tjian R. Isolation of cDNA encoding transcription factor $\mathrm{Sp} 1$ and functional analysis of the DNA binding domain. Cell. 1987; 51:1079-1090.

21. Wei D, Wang L, He Y, Xiong HQ, Abbruzzese JL, Xie K. Celecoxib inhibits vascular endothelial growth factor expression in and reduces angiogenesis and metastasis of human pancreatic cancer via suppression of $\mathrm{Sp} 1$ transcription factor activity. Cancer research. 2004; 64:2030-2038.

22. Takahra T, Smart DE, Oakley F, Mann DA. Induction of myofibroblast MMP-9 transcription in three-dimensional collagen I gel cultures: regulation by NF- $\mathrm{B}, \mathrm{AP}-1$ and $\mathrm{Sp} 1$. The international journal of biochemistry \& cell biology. 2004; 36:353-363.

23. Cornwell MM, Smith DE. SP1 activates the MDR1 promoter through one of two distinct G-rich regions that modulate promoter activity. Journal of Biological Chemistry. 1993; 268:19505-19511.

24. Gao Y, Jia Z, Kong X, Li Q, Chang DZ, Wei D, Le X, Suyun H, Huang S, Wang L, Xie K. Combining betulinic acid and mithramycin a effectively suppresses pancreatic cancer by inhibiting proliferation, invasion, and angiogenesis. Cancer research. 2011; 71:5182-5193.

25. Cornwell MM, Smith DE. SP1 activates the MDR1 promoter through one of two distinct G-rich regions that modulate promoter activity. The Journal of biological chemistry. 1993; 268:19505-19511.

26. Black AR, Black JD, Azizkhan-Clifford J. Sp1 and kruppellike factor family of transcription factors in cell growth regulation and cancer. Journal of cellular physiology. 2001; 188:143-160.

27. Kang DW, Lee SH, Yoon JW, Park WS, Choi KY, Min do S. Phospholipase D1 drives a positive feedback loop to reinforce the Wnt/beta-catenin/TCF signaling axis. Cancer Res. 2010; 70:4233-4242.

28. Choi HJ, Han JS. Overexpression of phospholipase D enhances Bcl-2 expression by activating STAT3 through independent activation of ERK and p38MAPK in HeLa cells. Biochim Biophys Acta. 2012; 1823:1082-1091.

29. Hu H, Hang JJ, Han T, Zhuo M, Jiao F, Wang LW. The M2 phenotype of tumor-associated macrophages in the stroma confers a poor prognosis in pancreatic cancer. Tumour biology. 2016.

30. Jiao F, Hu H, Yuan C, Wang L, Jiang W, Jin Z, Guo Z, Wang L. Elevated expression level of long noncoding RNA MALAT-1 facilitates cell growth, migration and invasion in pancreatic cancer. Oncology reports. 2014; 32:2485-2492. 